\title{
Outage-Constrained Beamforming for Two-Tier Massive MIMO Downlink with Pilot Reuse
}

\author{
Guozhen Xu, ${ }^{1}$ An Liu, ${ }^{2}$ Wei Jiang, ${ }^{1}$ Haige Xiang, ${ }^{1}$ and Wu Luo ${ }^{1}$ \\ ${ }^{1}$ The State Key Laboratory of Advanced Optical Communication Systems and Networks, Peking University, Beijing 100871, China \\ ${ }^{2}$ Department of Electrical and Computer Engineering, Hong Kong University of Science and Technology, Hong Kong
}

Correspondence should be addressed to Wei Jiang; jiangwei@pku.edu.cn

Received 20 August 2014; Revised 14 February 2015; Accepted 22 February 2015

Academic Editor: Ahmed T. Mobashsher

Copyright (c) 2015 Guozhen Xu et al. This is an open access article distributed under the Creative Commons Attribution License, which permits unrestricted use, distribution, and reproduction in any medium, provided the original work is properly cited.

\begin{abstract}
Massive multiple-input multiple-output (MIMO) systems and small cell networks are both regarded as promising candidates to meet the exponential growth of mobile data traffic for the next generation (5G) wireless communications. Hence, a new kind of multitier networks which combine massive MIMO macro cells with a secondary tier of small cells is proposed to resolve the contradiction of large network coverage and high data rate. In such multitier networks, it is inevitable to allocate nonorthogonal uplink pilot sequences to user equipment (UE) due to the large number of users. We propose a pilot reuse scheme by exploiting the unique architecture of this networks and analyse the special mixed channel state information (CSI) yielded by the pilot reuse scheme. Based on the mixed CSI, we formulate a downlink transmit beamforming problem of minimizing the total power consumption while satisfying the quality of service (QoS) requirements with outage constraints. After decomposing the original problem into simpler subproblems, we provide an efficient algorithm to combine these subproblems and solve them iteratively for generating the beamforming vectors. Monte Carlo simulations show that the average power consumption of the proposed pilot reuse scheme and its associated beamforming algorithm is close to that of the perfect CSI case.
\end{abstract}

\section{Introduction}

With the advent of the fifth generation (5G) cellular wireless communications, an ever-increasing demand of substantially higher throughput is a quite pressing task laid in front of us and drives the researchers to find new technology for wireless communications. Massive MIMO is a potential technology for meeting this demand and also taken as an attractive solution for $5 \mathrm{G}$ systems [1]. By implementing a large number of antennas at the BS, massive MIMO systems offer a high spatial and multiplexing resolution which can drastically improve the communication systems' performance in terms of data rate and reliability [2]. However, a critical issue for such massive MIMO systems is the excessive energy cost for their large number of antennas. How to provide higher data rates with lower energy consumption for such massive MIMO systems is a critical problem in front of us.

An innovative solution to this problem is a densified network with a multitier network architecture as stated in [3]. Sometimes, such multilayer and multiarchitecture networks are also called heterogeneous networks (HetNets). In [4], the authors have made further discussions about two-tier massive MIMO networks. The fundamental architecture of such networks is based on a deployment of a macro cell with very large antenna arrays in combination with a secondary tier of small cells (SCs) with a few antennas each. Macro cells are deployed for the coverage of large areas and capable of handling low data traffic or the users with a relative high mobility, and SCs with a reduced coverage range of tens of meters are designed for providing localized higher data rate communications.

However, there still exist some implicit obstacles to put such two-tier massive MIMO networks into practice. One problem is how to fulfill the channel estimation. In time division duplexing (TDD) cellular systems, channel estimation is obtained via the uplink training based on the channel reciprocity property. In other words, each user in the cell would be assigned a specific pilot sequence and these pilot sequences would be transmitted to the base station (BS) via the uplink. Acquiring perfect CSI inherently asks for 
sufficient numbers of orthogonal pilot sequences, which may not be possible for massive MIMO cellular systems. Hence, the pilot reuse is inevitable in massive MIMO systems, and the imperfect uplink training due to reusing the same pilot sequences tends to be a critical problem (referred to as "pilot contamination" problem [5]).

It is critical to design an appropriate pilot reuse scheme for mitigating the interference due to the pilot contamination. In [6-8], a time-shifted pilot reuse scheme was proposed to combat pilot contamination by symmetrically rearranging the uplink pilot transmission order for different cells and the system performance for such scheme with zeroforcing beamforming and a large number of BS antennas is also studied. Reference [9] studied the optimal pilot reuse factor for sum-rate maximization in massive MIMO systems. In [10], the authors proposed a pilot reuse scheme in homogenous multicell networks based on the degree of the spatial orthogonality in greedy fashion. In [11], the authors partitioned each cell into 3 sectors and assigned the reused pilot sequences in a symmetrical way in order to perform pilot contamination precoding (PCP). Recently, a novel pilot reuse scheme is proposed in [12] which exploits the channel spatial localization property to reduce the number of orthogonal pilots for uplink channel estimation in single cell massive MIMO systems. However, the pilot reuse scheme in [12] requires restrictive assumptions such as high channel spatial correlation and uniform linear array at the BS. In summary, all the works mentioned above focused on either homogenous multicell networks or single cell systems. As a result, they cannot be directly applied to (or are not optimized for) HetNets with asymmetrical system architecture.

Another problem is how to tackle the intercell interference. With limited spectral resources, a cochannel deployment of macro cell and secondary tier SCs is the only viable solution, and this in turn requires a sophisticated interference management scheme across the tiers. The crosstier interference is even thought to be one of the bottlenecks for designing high-performance HetNets [13].

Now, a tough problem of proposing a high-performance and low-complexity interference management scheme to guarantee the system's QoS requirements with limited uplink pilot resources is in front of us.

For this problem, robust transmit beamforming methods which take the CSI errors into consideration are in need for the reason that "pilot contamination" causes imperfect CSI. In $[14,15]$, a sum-rate maximization problem with imperfect CSI was investigated. However, these methods mainly focused on the worst case approach under the assumption of normbounded uncertainty which is not suitable for the considered system. Furthermore, a more efficient conservative formulation is presented which involves solving a semidefinite programming (SDP) in [16]. By using the Bernstein-type inequality, a chance constrained beamforming problem in cognitive radio networks is also provided in [17]. The kernel of these methods is to transform the original problem to a tractable SDP problem conservatively. However, the huge computation complexity of SDP, $O\left(n^{6.5}\right)$, makes these methods unattractive in massive MIMO systems [18].

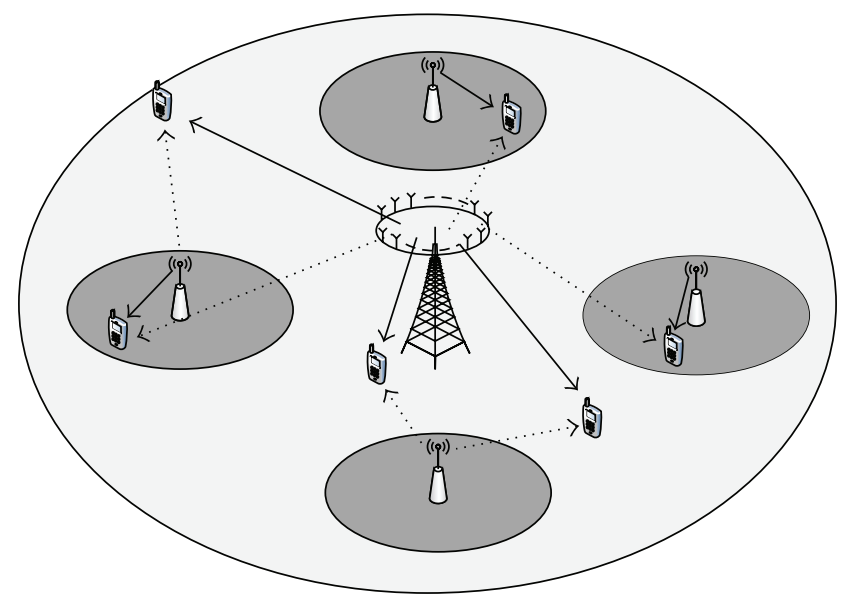

FIgURE 1: One typical architecture of a two-tier network. A macro cell tier is overlaid with a secondary tier of small cells. The interfering signals are marked by dotted lines and the intended signals are marked by solid lines.

Compared with the previous works, we propose a pilot reuse scheme by exploiting the unique architecture of this HetNet. A "mixed CSI" is achieved at the BS due to our pilot reuse scheme. Based on the mixed CSI, we formulate the energy-efficient beamforming problem as a chance constraint programming and decompose it into simpler subproblems. Then, an efficient iterative algorithm is provided to combine these subproblems and solve them iteratively for obtaining the beamforming vectors.

The rest of this paper is organized as follows. In Section 2, the system model is provided. How to compress the number of the uplink pilot sequences and how to design the energyefficient beamformer with the mixed CSI are presented and solved in Section 3. Section 4 presents the numerical results and Section 5 concludes this paper.

\section{System Model}

We consider the downlink of a TDD orthogonal frequency division multiplexing (OFDM) system. Different from the traditional systems with single base station, one macro base station (MBS) and $S$ low range small cell base stations (SBSs) separately serve their intended single antenna users in the same frequency band shown as in Figure 1. The MBS has $N_{\text {BS }}$ antennas and each SBS has $N_{\text {SC }}$ antennas, where $N_{\text {BS }}$ is assumed to be a large number which is known as massive MIMO. We consider that the total number of the served users is $K$; since the total number of transmit antennas is $N=N_{\mathrm{BS}}+$ $S N_{\mathrm{SC}}$, the downlink channel propagation matrix between the $N$ antennas and $K$ users can be described by a $K \times N$ matrix, $\mathbf{H}$. The base stations can exploit channel reciprocity to obtain channel state information at the transmitter (CSIT) based on uplink training. Here we denote the macro cell as cell 0 and the $j$ th SC is denoted as cell $j$. The channel of the $k$ th user is represented as $\mathbf{h}_{k}=\left[\begin{array}{llll}\mathbf{h}_{0, k} & \mathbf{h}_{1, k} & \cdots & \mathbf{h}_{S, k}\end{array}\right] \in \mathbb{C}^{1 \times N}$, where $\mathbf{h}_{0, k} \in \mathbb{C}^{1 \times N_{\mathrm{BS}}}$ and $\mathbf{h}_{j, k} \in \mathbb{C}^{1 \times N_{\mathrm{SC}}}, j=1, \ldots, S$. Let $h_{j, n, k} \in \mathbb{C}, \forall j, n, k$, denote the entries of $\mathbf{h}_{j, k}, \forall j, k$, and 
$h_{j, n, k}$ describes the channel between the $n$th antenna in cell $j$ and the $k$ th user. We consider a composite fading channel; that is, $h_{j, n, k}=\sqrt{d_{j, n, k}} z_{j, n, k}$, where $d_{j, n, k}$ is the path gain between antenna $n$ in cell $j$ and user $k$, and $z_{j, n, k} \in \mathbb{C}$ is the small scale fading with i.i.d. $\sim \mathscr{C} \mathscr{N}(0,1)$ entry. Here, we assume a time block fading model. Thus path fading vectors $d_{j, n, k}, \forall j, n, k$, stay constant during each coherence interval and these vectors are assumed to be independent in different coherence blocks. Since all the transmit antennas of MBS are considered to be center collected, $\mathbf{h}_{0, k} \sim \mathscr{C} \mathscr{N}\left(\mathbf{0}, d_{0, k} \mathbf{I}\right)$ is satisfied, where $d_{0, k}, \forall k=1, \ldots, K$, are independent random variables describing the path loss fading.

We divide the $K$ users into two groups represented by small-cell user equipment (SUE) and macro cell user equipment (MUE). If user $k$ is located in the coverage of the $j$ th SC and register at the $j$ th SBS for a certain high quality communication service, then user $k$ is a SUE of SBS $j$. The other group is the MUE who do not register at any SC and communicate with the MBS for a common quality communication service. To specify which cell it belongs to, the SUE $k$ located in the $j$ th SBS can also be denoted as user $k_{j}$. If user $k$ is a MUE, then it can be also denoted as $k_{0}$ similarly. For convenience, we suppose that each SC has $N_{s}$ registered SUE. Thus, there are $N_{s} S \leq K$ users registered at all the SCs totally.

We assume that the MBS and SBSs are connected to a backhaul network with limited capacity which enables interference coordination. When the backhaul is capacity limited, the coordination strategy with no data and only limited CSI sharing is preferred via the backhaul [19]. Note that each user is considered to be served only by its associated BS in our system; that is, MUE are served by MBS and each SUE is served by its associated SBS. The information symbols for user $k_{j}$ from its associated $j$ th BS can be denoted as $x_{k_{j}}$ (or $x_{k}$ ). These information symbols are independently precoded by the beamforming vectors, $\mathbf{w}_{0, k} \in \mathbb{C}^{N_{\mathrm{BS}}}$ or $\mathbf{w}_{j, k} \in \mathbb{C}^{N_{\mathrm{SC}}}$, where $\mathbf{w}_{j, k}$ is the beamforming vector at the $j$ th BS for the $k$ th user. Then the received signal at user $k$ is (note that $\mathbf{w}_{j, k}=\mathbf{0}$ satisfies when BS $j$ does not serve the $k$ th user)

$$
y_{k_{j}}=\mathbf{h}_{j, k_{j}} \mathbf{w}_{j, k_{j}} x_{k_{j}}+\underbrace{\sum_{i=1, i \neq k_{j}}^{K}\left(\sum_{s=0}^{S} \mathbf{h}_{s, k_{j}} \mathbf{w}_{s, i} x_{i}\right)}_{\text {interference }}+n_{k_{j}} \text {, }
$$

where the term $n_{k}$ (or $n_{k_{j}}$ ) is the circularly symmetric complex Gaussian receiver noise which is an i.i.d. $\mathscr{C} \mathscr{N}\left(0, \sigma_{k}^{2}\right)$ random variable. The signal $x_{k}$ is originated from independent Gaussian codebooks and constrained to have a total expected power of one; that is, $\mathbb{E}\left\{\left|x_{k}\right|^{2}\right\}=1, \forall k$.

For a given set of optimized beamforming vectors $\mathbf{w}_{j, k}, \forall j, k$, the signal-to-interference and noise ratio (SINR) of the $k$ th user can be expressed as

$$
\Gamma_{k_{j}}=\frac{\left|\mathbf{h}_{j, k_{j}} \mathbf{w}_{j, k_{j}}\right|^{2}}{\sum_{i=1, i \neq k_{j}}^{K}\left(\sum_{s=0}^{S}\left|\mathbf{h}_{s, k_{j}} \mathbf{w}_{s, i}\right|^{2}\right)+\sigma_{k_{j}}^{2}} .
$$

Consequently, the information rate of user $k$ is calculated by

$$
\mathscr{R}_{k}=\log _{2}\left(1+\Gamma_{k}\right) \text {. }
$$

We model the power consumption of the system as an addition of the transmit power and the circuit power like that in [20] as follows:

$$
P_{\text {tot }}=P_{t}+P_{s}
$$

where $P_{\text {tot }}$ denotes the total energy consumption of the system. $P_{t}$ is the dynamic power consumption known as the aggregation of the emitted power and $P_{s}$ is modeled as the static power consumption cost by the BS components attached to each antenna, for example, A/D (D/A) converters, filters, and mixers, where $P_{t}$ and $P_{s}$ can be calculated by

$$
\begin{aligned}
& P_{t}=\sum_{j=0}^{S} \eta_{j}^{-1} \sum_{k=1}^{K}\left\|\mathbf{w}_{j, k}\right\|_{F}^{2}, \\
& P_{s}=\rho_{0} N_{\mathrm{BS}}+\sum_{j=1}^{S} \rho_{j} N_{\mathrm{SC}},
\end{aligned}
$$

where $\eta_{j} \in(0,1]$ represents the efficiency of transmitter power amplifiers in the $j$ th cell, $\rho_{j}$ is the power consumption in the circuits of each antenna in the $j$ th cell, and $\|\cdot\|_{F}$ stands for the Frobenius norm.

\section{Pilot Reuse and Outage-Constrained Beamforming}

In this section, we will introduce our pilot reuse scheme and analyze the special mixed CSI yielded by this scheme. A low complexity algorithm for downlink beamforming is also provided based on the mixed CSI.

3.1. Pilot Reuse and System Operating. In such two-tier HetNet, at least $K$ orthogonal uplink pilot sequences are needed to achieve the global perfect CSI [21] (in this paper, we ignore the issues related to channel estimation and simply assume that the perfect CSI between one UE and certain BS is available at the BS if the UE's allocated pilot sequence is orthogonal to that of the other UEs'. Since pilot power and data power could be assigned individually [22, 23], this is an appropriate assumption even when the users' SINR is varying). In practice, the short channel coherence intervals do not allow for such long training sequences [24]. Hence, in such multi-BS networks, nonorthogonal training sequences must be utilized. The pilot contamination problem was studied in [5] which found that pilot contamination would result in corruption of precoding matrices and cause significant intercell interference (ICI). The ICI brings about the saturation effect; that is, the system throughput does not grow with the increasing of the number of BS antennas.

Compressing the number of pilot sequences to a precise value and allocating these pilots in an appropriate way is a hard problem. To the best of the authors' knowledge, there has been no systematic method for this task currently. While, by 


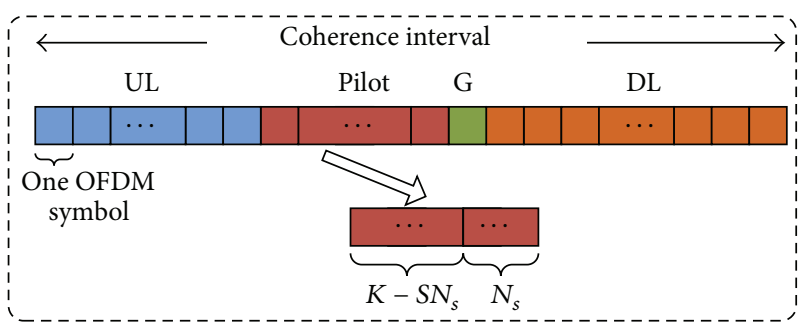

(a)

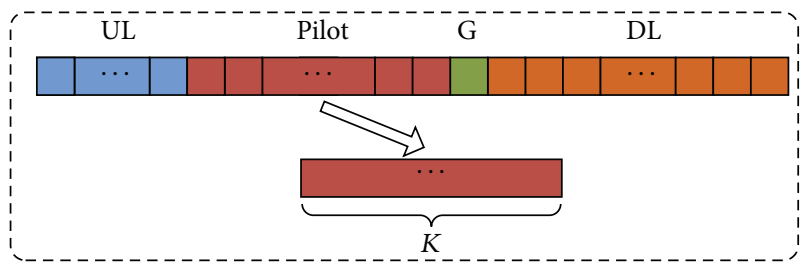

(b)

FIGURE 2: A kind of TDD-based frame structure. The pilot reuse scheme for the two-tier HetNet is presented in (a). The pilot scheme for achieving global perfect CSI is presented in (b).

exploiting the special architecture of the considered HetNet, a simplified scheme for this pilot allocation problem can be obtained. In such HetNet, the SCs are geometrically separated with each other and the coverage regions of different SCs are often nonoverlapped. As a result, the users in a SC hardly receive signal from other SBSs, which means that the interference among the SCs can be ignored. Thus, the crosstier interference is the bottleneck to improve the throughput of such HetNet.

Now, we consider how to compress the number of pilot sequences and allocate these pilots to different users in such HetNet. On one hand, the MUE may walk around the macro cell with a relative high mobility; instantaneous CSI is crucial for the MBS to serve these MUE; on the other hand, each SC would have strong channel gain to its associated SUE, instantaneous CSI is also in need for this SBS and its associated SUE. Hence, we allocate orthogonal pilot sequence to each MUE and all the SCs share the same $N_{s}$ pilot sequences for their $N_{s}$ active users. Considering a TDD frame structure similar to that in [24], we propose the frame structure of this HetNet in Figure 2(a).

For comparison, the frame structure for achieving the global perfect CSI is presented in Figure 2(b). When global perfect CSI is preferred, at least $K$ orthogonal pilot sequences are in need in one TDD frame. This would be intolerable for the considered macro cell massive MIMO systems in which the number of users would be large.

Now, we pay attention to the special kind of CSI achieved by our proposed pilot reuse scheme. For any MUE, it will transmit its nonreused pilots to the BSs in the uplink training phase. Both the MBS and the SBSs can receive the pilot signal and then estimate the channels between this MUE and the BSs. Since all the pilot sequences of MUE are nonreused, both the MBS and the SBSs could have the perfect CSI while, for a SUE $k_{j}$, it will transmit its reused pilots to the BSs in the

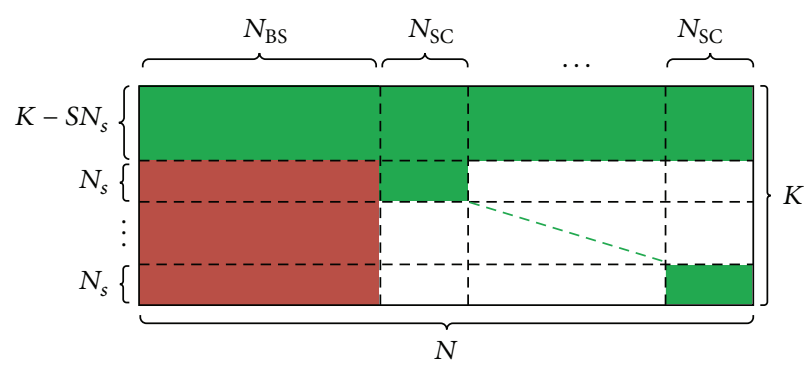

Figure 3: The rearranged $\mathbf{H}$ for the two-tier network.

uplink training phase. Since the coverage regions of different SCs are nonoverlapped and the receive antenna gain of MBS is usually much larger than the SBS, it means that the minor channel coefficients between SUE $k_{j}$ and other SCs can be ignored, and only the $j$ th SBS and the MBS can receive the pilot signal. Then the $j$ th SBS and the MBS can estimate the channels between SUE $k_{j}$ and them. Note that SBS $j$ cannot receive this pilot sequence from the SUE of other SCs, while the $\mathrm{MBS}$ receive this pilot sequence from all the SCs. It means that the $j$ th SBS will achieve the perfect CSI of SUE $k_{j}$, and the MBS (M) could just obtain the statistical knowledge of this SUE, for example, its path loss fading.

For simplicity, we rearrange the original channel matrix $\mathbf{H}$ as in Figure 3, where the first $N_{\mathrm{BS}}$ columns correspond to the channel matrix of the MBS antennas and the following $N_{\mathrm{SC}}$ columns correspond to the channel matrix of the antennas belonging to the 1st SBS and so on. It is similar that the first $K-S N_{s}$ rows correspond to channel matrix of the MUE and the next $N_{s}$ rows correspond to the channel matrix of the SUE in the 1st SC and so on.

Besides, we use different colours to distinguish the different kinds of CSI. The perfectly known CSI is marked in green and the contaminated CSI is marked in red. The statistical knowledge of these contaminated CSI is assumed to be known at the BS, for example, the path loss fading of these users. The other space of the channel matrix is marked in white and stands for the minor channel coefficients which can be ignored. We call such kind of CSI as the "mixed CSI" for containing different kinds of CSI.

3.2. Outage-Constrained Beamforming with Mixed CSI. The obtained mixed CSI, that is, some entries of $\mathbf{H}$ are perfect known at the BS while the others are statistically known or 0 , brings a new technical challenge for the system downlink beamforming. Since some CSI is unknown, the QoS outage is inevitable. To guarantee the QoS and its outage, we impose a probabilistic QoS constraint as follows:

$$
\operatorname{Pr}\left(\Gamma_{k}>\gamma_{k}\right) \geq 1-\delta_{k}, \quad \forall k
$$

where $0<\delta_{k}<1$ indicates that the system should guarantee the QoS requirements for the $k$ th users with probability of at least $1-\delta_{k}$. Then, we can formulate the energy-efficient beamforming problem as a problem of minimizing the total 
transmit power while satisfying the system chance constraint as

$$
\begin{aligned}
\mathscr{P}_{0}: \min _{\mathbf{w}_{j, k}} & P_{\text {tot }} \\
\text { s.t. } & \operatorname{Pr}\left(\Gamma_{k}>\gamma_{k}\right) \geq 1-\delta_{k}, \quad \forall k .
\end{aligned}
$$

Recall that there are two different groups of users, that is, MUE and SUE. Hence, we can denote $\mathscr{M} \triangleq\left\{1, \ldots, K-S N_{s}\right\}$ as the set of the MUE and let $\mathcal{S} \triangleq\left\{K-S N_{s}+1, \ldots, K\right\}$ stand for the set of all the SUE for convenience. Moreover, there are also two types of QoS constraints for such two types of users. As for the MUE, both the MBS and SBSs can obtain their perfect CSI. It means that the type of QoS for the MUE should be determinate; that is, $\delta_{k}=0, \forall k \in$ $\mathscr{M}$. However, for the SUE, there is uncertainty in their CSI causing occasional QoS outage. Thus, the type of QoS for the SUE should be probabilistic; that is, it should involve outage probability constraint. Hence, the chance constrained beamforming with the achievable mixed CSI is formulated as $\mathscr{P}_{1}$ :

$$
\begin{aligned}
\mathscr{P}_{1}: \min _{\mathbf{w}_{j, k}} P_{\text {tot }} \\
\text { s.t. } \quad \operatorname{Pr}\left(\Gamma_{k} \geq \gamma_{k}\right)>1-\delta_{k}, \quad \forall k \in \mathcal{S}, \\
\Gamma_{k} \geq \gamma_{k}, \quad \forall k \in \mathscr{M} .
\end{aligned}
$$

$\mathscr{P}_{1}$ is to optimize the beamforming vectors $\left\{\mathbf{w}_{j, k}, \forall k\right\}$ for a total transmit power minimization. It aims to ensure that each MUE $k$ is served with a minimal information rate of $\log _{2}\left(1+\gamma_{k}\right)$ and each SUE $k$ is served with a minimal information rate of $\log _{2}\left(1+\gamma_{k}\right)$ with an outage probability no greater than $\delta_{k}$.

Usually, by using semidefinite relaxation (SDR), $\mathscr{P}_{1}$ can be conservative transformed to a SDP. However, time complexity of standard interior-point SDP solvers could be as high as $O\left(n^{6.5}\right)$. Such intensive computation cost may prohibit SDR applicable to massive MIMO systems. Moreover, the conservatism of such SDR method is also hard to control.

In order to overcome this difficulty, we first decompose the complex problem $\mathscr{P}_{1}$ into simpler subproblems and then propose an efficient iterative algorithm to combine these subproblems and solve $\mathscr{P}_{1}$ iteratively.

As for the MUE, only the MBS transmits information symbols to them, and their beamforming vectors are generated by solving the following convex problem:

$$
\begin{aligned}
& \mathscr{P}_{s 0}: \\
& \min _{\mathbf{w}_{0, k}}\left\|\mathbf{w}_{0, k}\right\|^{2} \\
& \text { s.t. } \quad \frac{\left|\mathbf{h}_{0, k} \mathbf{w}_{0, k}\right|^{2}}{\sum_{i \in \mathscr{M}, i \neq k}\left|\mathbf{h}_{0, k} \mathbf{w}_{0, i}\right|^{2}+\sum_{i \in \mathcal{S}}\left(\sum_{s=1}^{S}\left|\mathbf{h}_{s, k} \mathbf{w}_{s, i_{s}}\right|^{2}\right)+\sigma_{k}^{2}} \\
& \\
& \quad>\gamma_{k}, \quad \forall k \in \mathscr{M} .
\end{aligned}
$$

As for the SUE, since one SUE in a SC hardly receives signal from other SBSs, the problem of optimizing their beamforming vectors could be simplified as follows:

$$
\begin{aligned}
& \mathscr{P}_{s 1}: \min _{\mathbf{w}_{j, k_{j}}}\left\|\mathbf{w}_{j, k_{j}}\right\|^{2} \\
& \begin{array}{cl}
\text { s.t. } & \frac{\left|\mathbf{h}_{j, k_{j}} \mathbf{w}_{j, k_{j}}\right|^{2}}{\sum_{i \in \mathscr{M}}\left|\mathbf{h}_{0, k_{j}} \mathbf{w}_{0, i}\right|^{2}+\sum_{i \in \mathcal{S}, i \neq k_{j}}\left|\mathbf{h}_{j, k_{j}} \mathbf{w}_{j, i}\right|^{2}+\sigma_{k_{j}}^{2}} \\
>\gamma_{k_{j}}, \quad \forall k_{j} \in \mathcal{S} .
\end{array}
\end{aligned}
$$

From $\mathscr{P}_{s 0}$ and $\mathscr{P}_{s 1}$, we find that the cross-tier interference of SUE and MUE is in relation with each other; that is, it needs the knowledge of the MUE beamforming vectors to calculate the interference power of SUE, and vice versa.

Now we are ready to combine $\mathscr{P}_{s 0}$ with $\mathscr{P}_{s 1}$ and use them to solve $\mathscr{P}_{1}$ iteratively. For convenience, the cross-tier interference of the $k$ th user caused by SBSs or MBS is denoted as $I_{s_{k}}$ and $I_{m_{k}}$, respectively, where

$$
\begin{gathered}
I_{s_{k}}=\sum_{i \in \mathcal{S}}\left(\sum_{s=1}^{S}\left|\mathbf{h}_{s, k} \mathbf{w}_{s, i}\right|^{2}\right), \quad k \in \mathscr{M}, \\
I_{m_{k}}=\sum_{i \in \mathscr{M}}\left|\mathbf{h}_{0, k} \mathbf{w}_{0, i}\right|^{2}, \quad k \in \mathcal{S} .
\end{gathered}
$$

Considering the initial interference caused by the SBS and MBS as 0 , then the optimization problem $\mathscr{P}_{s 1}$ can be independently solved by each SBS and the beamforming vectors of $\mathbf{w}_{j, k_{j}}, \forall j \neq 0$, can be obtained. Since the CSI between MUE and all SBSs is perfectly known, MBS can broadcast $\mathbf{h}_{s, k}, \forall s \neq 0, k \in \mathscr{M}$, to each associated SBS beforehand. Hence, the $j$ th SBS could compute the cross-tier interference it causes by calculating by

$$
I_{s_{j, k}}=\sum_{i \in \mathcal{S}}\left(\left|\mathbf{h}_{j, k} \mathbf{w}_{j, i}\right|^{2}\right), \quad k \in \mathscr{M}
$$

and transfer it to the MBS.

After getting the total cross-tier interference $I_{s_{k}}=$ $\sum_{j=1}^{S} I_{s_{j, k}}$, the MBS can generate its beamforming vectors by solving $\mathscr{P}_{s 0}$. Since the CSI between MBS and SUE is statistically known, with the knowledge of $\left\{\mathbf{w}_{0, k}, k \in \mathscr{M}\right\}$, the cross-tier interference caused by the MBS to the $k$ th SUE can be calculated as

$$
I_{k}=d_{0, k} F_{Y}^{-1}\left(1-\delta_{k}\right)
$$

where

$$
F_{Y}(y)=1-\sum_{1}^{m} \frac{\lambda_{i}^{m-1}}{\prod_{l \neq i}\left(\lambda_{i}-\lambda_{l}\right)} e^{-y / \lambda_{i}},
$$

with $\lambda_{i}, i=1, \ldots, m$ being the nonzero eigenvalues of $\left(\sum_{i \in \mathscr{M}} \mathbf{w}_{0, i} \mathbf{w}_{0, i}^{H}\right)$ in descending order (a detailed derivation of (13) and (14) can be found in the appendix). 


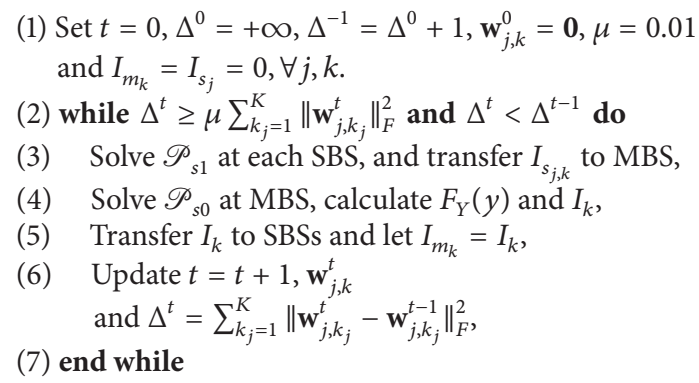

Algorithm 1

Equation (13) means that $\operatorname{Pr}\left(I_{k} \geq I_{m_{k}}\right) \geq 1-\delta_{k}$; that is, the cross-tier interference caused by the MBS to the $k$ th SUE will be larger than $I_{k}$ with a probability no greater than $\delta_{k}$.

After the MBS broadcasting $\left\{I_{k_{j}}, k_{j} \in \delta\right\}$ to the $j$ th SBSs to update their local $I_{m_{k}}$, SBSs repeat the process of solving the problem $\mathscr{P}_{s 1}$ mentioned above with the updated $I_{m_{k}}$. Such iterative process will stop until the predefined stop criterion is met.

Summarizing the above steps, we thus obtain the distributed algorithm as in Algorithm 1, where $\Delta^{t}$ denotes the difference between the beamforming matrices in two successive iterations and $\mu$ is utilized to constrain the maximum of this difference. The stop criterion means that the iterative process is permissible only when the following two conditions are satisfied simultaneously:

(i) $\Delta^{t}$ is not smaller than a ratio of the total transmit power;

(ii) $\Delta^{t}$ is shrinking.

Since $\mathscr{P}_{s 0}$ and $\mathscr{P}_{s 1}$ are both second-order cone programming problems (SOCPs) [25], they can be efficiently handled by standard solvers, for example, SeDuMi [26], and the computation complexity is about $O\left(N_{\mathrm{BS}}^{3.5}\right)$ and $O\left(N_{\mathrm{SC}}^{3.5}\right)$, respectively [18].

Remark 1. In Algorithm 1, it requires exchanging the knowledge of cross-tier interference between MBS and SBSs. Note that, in the process of exchanging interference knowledge, only one real value is transferred via the backhaul each time, which results in a total signaling of $S N_{S}+S\left(K-S N_{S}\right)$ real values per iteration. Some other operations would also affect this total signaling. For example, if SUE broadcast their $\left\{\mathbf{w}_{j, i}, \forall j \neq 0, i \in \mathcal{S}\right\}$ to MBS instead of transferring $I_{s_{j, k}}$, the total signaling would be $S N_{s}+2 S N_{s} N_{S C}$. Hence, mobile operators should choose an appropriate kind knowledge to exchange so as to yield a minimum backhaul signaling based on the architecture of the HetNet.

Remark 2. It is difficult to bound the exact number of iterations required by the proposed algorithm. However, simulations show that the proposed algorithm always converges within a few number of iterations. For example, in all of our Monte Carlo simulations (over 200,000 random channel realizations), the maximum number of iterations is 25 . Most of the time, the proposed algorithm will converge within 5 times of iterations (see Section 4 for details).

\section{Simulation Results}

Monte Carlo simulations are performed to verify the conclusions and testify the performance of the proposed pilot reuse scheme and its associated downlink beamforming algorithm. The system performance is evaluated in terms of average total power consumption. When keeping the QoS constraints constant, the energy efficiency of the system is inversely proportional to its average total power consumption.

4.1. Simulation Settings. The simulation parameters are set as follows. We consider a circular cell with one MBS and $S=4$ small cells. The radii of the macro cell and small cell are $R=0.5 \mathrm{~km}$ and $r=40 \mathrm{~m}$, respectively. Since the user assignment problem is independent of the beamforming problem considered in this paper, the users in the whole systems are scheduled in a round robin fashion for fairness consideration (please refer to [27-29] and the references therein for more about the user assignment problem). The goal of fairness user scheduling in this paper is to make the system operate at some point of its ergodic achievable rate region so as to provide useful insights into the impact of model parameters (e.g., number of antennas and outage probability constraint). We set the following: there are $K=10$ active users in the whole system, where 6 users are uniformly distributed in the macro cell and each SC has one user uniformly distributed within its small cell coverage. We evaluate the average power consumption performance over user locations and channel realizations. The position of the MBS is set as $(0,0)$ and the overlaid SBSs are located at $(0.5 R, 0.5 R),(-0.5 R, 0.5 R)$, $(0.5 R,-0.5 R)$, and $(-0.5 R,-0.5 R)$, respectively. Though there is no definite standard of the future $5 \mathrm{G}$ network, it is commonly thought that the future $5 \mathrm{G}$ network is an evolution of the current communication network (3G/4G network) [30]. Hence, we take some system parameters of the current communication network for simulation, and the channel model is assumed as follows: (i) there are 600 subcarriers in the $10 \mathrm{MHz}$ total system bandwidth, where the bandwidth of each subcarrier is $15 \mathrm{KHz}$; (ii) the channel between the MBS and UEs is using the 3GPP LTE pathloss mode: PL = $148.1+37.6 \log _{10}(d)($ in $\mathrm{dB}$ ) with $d$ (in $\mathrm{km}$ ) being the MBSUE distance; (iii) within each SC, the channel is modeled as $\mathrm{PL}=127+30 \log _{10}(d)($ in $\mathrm{dB})$ with $d$ (in $\mathrm{km}$ ) being the SBSUE distance; (iv) the energy consumption parameters are set according to [31], that is, $\eta_{0}=0.388, \eta_{j}=0.052, \forall j \neq 0$, $\rho_{0}=189 \mathrm{~mW}$, and $\rho_{j}=5.6 \mathrm{~mW}, \forall j \neq 0$; and (v) the noise covariance of receivers is set as $-127 \mathrm{dBm}$.

4.2. Comparison with the Perfect CSI Case. It would be interesting to simulate the performance gap between the proposed low complexity algorithm and the optimal beamforming method with chance constraint. However, to the best of the authors' knowledge, there has not been such optimal methods in existing work. Thus, we propose to compare Algorithm 1 


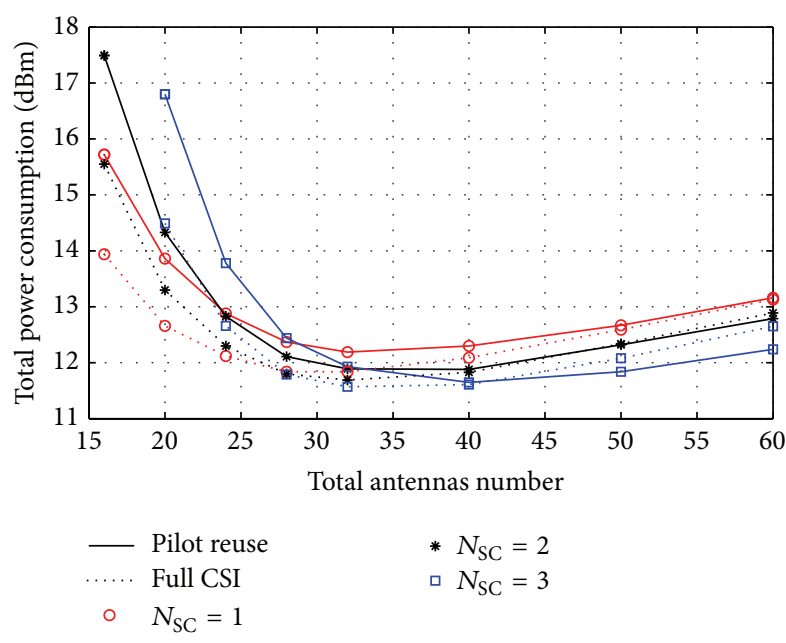

FIGURE 4: The average power consumption of the whole system with different numbers of $N$ and $N_{\mathrm{SC}}$.

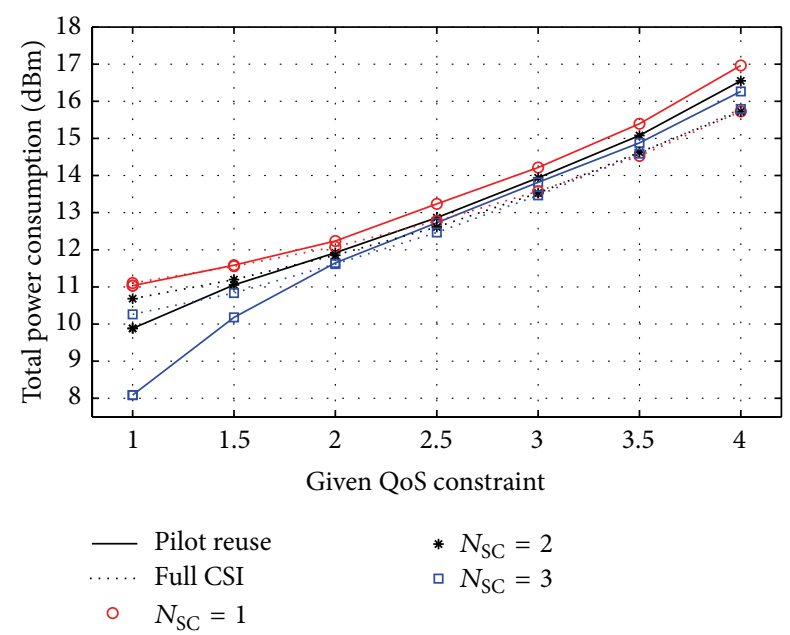

FIGURE 5: The average power consumption of the whole cell for different QoS constraints. The QoS constraints are measured in bps/Hz per user.

with the following baseline which is labeled as "full CSI" in Figures 4 and 5. In this baseline, we assume that the fully coordinated MBS and SBSs are able to share both the data and channel information. Then CoMP-JP can be used to convert the intercell interference into useful signal [32]. In other words, this information is available at all BSs, who share their antennas together to serve the users jointly. If the beamforming method in CoMP-JP is optimal, this baseline provides a strictly upper bound for the maximum sum-rate. Since there exists QoS outage constraint in Algorithm 1 which may be helpful to save some transmit power, this baseline is likely to be the upper bound of Algorithm 1 especially when $\delta_{k}$ is tiny.

Now, we introduce the beamforming method for CoMPJP. In this condition, the problem of minimizing the total power consumption while satisfying the QoS constraints is formulated as follows:

$$
\begin{array}{ll}
\min _{\mathbf{w}_{k}} & P_{\text {tot }} \\
\text { s.t. } & \Gamma_{k} \geq \gamma_{k}, \quad \forall k,
\end{array}
$$

where $\Gamma_{k}$ is shown as

$$
\Gamma_{k}=\frac{\left|\mathbf{h}_{k} \mathbf{w}_{k}\right|^{2}}{\sum_{i \neq k}\left|\mathbf{h}_{k} \mathbf{w}_{i}\right|^{2}+\sigma_{k}^{2}},
$$

with $\mathbf{h}_{k}=\left[\begin{array}{llll}\mathbf{h}_{0, k} & \mathbf{h}_{1, k} & \cdots & \mathbf{h}_{S, k}\end{array}\right] \in \mathbb{C}^{1 \times N}$ and $\mathbf{w}_{k}=$ $\left[\begin{array}{llll}\mathbf{w}_{0, k}^{H} & \mathbf{w}_{1, k}^{H} & \cdots & \mathbf{w}_{S, k}^{H}\end{array}\right]^{H} \in \mathbb{C}^{N}$.

After applying SDR by dropping the rank constraints, we can relax (15) to a semidefinite optimization problem on standard form (see details in [33, Chapter 18])

$$
\begin{array}{ll}
\min _{\mathbf{W}_{k}} & P_{\text {tot }} \\
\text { s.t. } & \mathbf{h}_{k}\left(\left(1+\gamma_{k}^{-1}\right) \mathbf{W}_{k}-\sum_{i=1}^{K} \mathbf{W}_{i}\right) \mathbf{h}_{k}^{H} \geq \sigma_{k}^{2}, \quad \forall k, \\
& \mathbf{W}_{k} \geq \mathbf{0}, \quad \forall k,
\end{array}
$$

where $\mathbf{W}_{k}$ is positive semidefinite with $\mathbf{W}_{k}=\mathbf{w}_{k} \mathbf{w}_{k}^{H}$.

It can be proved that these rank constraints can be relaxed without losing optimality [33, Chapter 18]. It means that the original problem (15) can be solved as a convex optimization problem (17).

4.3. Performance of the Proposed Algorithm. In this part, we will present the performance of the proposed algorithm and compare it with the optimal COMP-JP method mentioned above.

First of all, we focus on the impact of the different numbers of antennas at the MBS and SBS. Figure 4 compares the power consumption of the full CSI scenario (or perfect CSI scenario) and the pilot reuse scenario with different numbers of antennas. The solid lines show the case when the full downlink CSI is available at the BSs, while the dotted lines depict the one when mixed CSI is available at BSs. The given QoS requirement of each user is set as $\left\{\gamma_{k}=3, \forall k\right\}$ and the QoS outage probability requirement for the mixed CSI scenario is set to be $\delta_{k}=0.1, \forall k \in \delta$.

In the mixed CSI scenario, when $N_{\mathrm{SC}}$ is fixed, it will be more energy efficient when $N$ is not too large or small. This is because that when $N$ is not too large, the transmit power is much larger than the circuit power consumption, and the consumed power is mainly provided for the data transmission. However, with the increasing of $N$, when the circuit power consumption is comparable to or even dominates the transmit power, too much additional circuit power is consumed by the large number of active antennas and therefore decrease the energy efficiency. In other words, appropriate number of active antennas could improve the energy efficiency of the system. It has the same phenomenon in the full CSI scenario. 
Then, we focus on the impact of the number of antennas at each SBS, $N_{\mathrm{SC}}$. When $N$ is not so large, the energy efficiency decreases with the increasing of $N_{\mathrm{SC}}$, while a larger $N_{\mathrm{SC}}$ will yield a higher energy efficiency when $N$ is large enough. It tells us that, when $N$ is not large enough, it is not favorable to extract antennas from the macro cell and settle them in the SCs which will leave not enough macro cell antennas to handle the MUE. When $N$ is large enough which is sufficient for the MUE, appropriate extraction antennas from the MBS to the SCs will not only improve the throughput performance of SBSs but also decrease circuit power consumption of the MBS.

Besides, we find that the power consumed by the proposed algorithm is larger than that the full CSI scenario when the number of $N$ is small. With the increasing of $N$, the proposed algorithm is likely to consume less power than the full CSI scenario. This interesting phenomenon is mainly due to the fact the full CSI scenario and the proposed scheme have different QoS requirements. In the full CSI scenario, the SINR constraint in (15) must be satisfied for all channel realizations. On the other hand, in the proposed scheme, SINR outage is allowed; that is, the SINR constraint in (8) is satisfied with probability at least $1-\delta_{k}$. Hence, the QoS requirement for the full CSI scenario is more stringent, which may lead to larger transmit power than that of the proposed scheme.

Moreover, we find that, when choosing the most energy efficient $N$ (about 35), the average energy consumption of the proposed pilot reuse scheme is larger than that of the full CSI scenario, and the energy consumption gap of them is no larger than $0.3 \mathrm{~dB}$. Though consuming some extra energy than the full CSI scenario, our scheme will greatly compress the number of pilot sequences especially for those highly densified network with large number of small cells.

Hence, we summarize that choosing a suitable number of the total antennas and allocating an appropriate portion of them to the SCs would intensively enhance the energy efficiency of the whole system and the proposed algorithm is especially fit for the massive MIMO systems.

Figure 5 compares the average power consumption for different QoS constraints in the full CSI scenario and the mixed CSI scenario when $N=40$ and $\delta_{k}=0.1, \forall k \in \mathcal{S}$. We observe that, when the required QoS constraints are not large, the systems with larger $N_{\mathrm{SC}}$ consume less power even than the full CSI cases. This is for the reason that $\delta_{k}$ will relax the QoS requirements to some extent and save some energy consequently while, with the increasing of the required QoS constraints, the gap of the power consumption between the full CSI scenario and the mixed CSI scenario tends to be static, which ensures that the proposed algorithm is widely adaptive to different QoS requirements.

Figure 6 shows the impact of the QoS outage probability constraints on the power consumption when $N=40$ and $\gamma_{k}=3, \forall k$. We find that when decreasing $\delta_{k}$ from 0.2 to 0.01 it consumes more power. However, when $N_{\mathrm{SC}}$ is fixed, the difference of power consumption is not evident. Hence, we conclude that, over a reasonable range of QoS outage probability, the consumed power of our scheme is likely to be static.

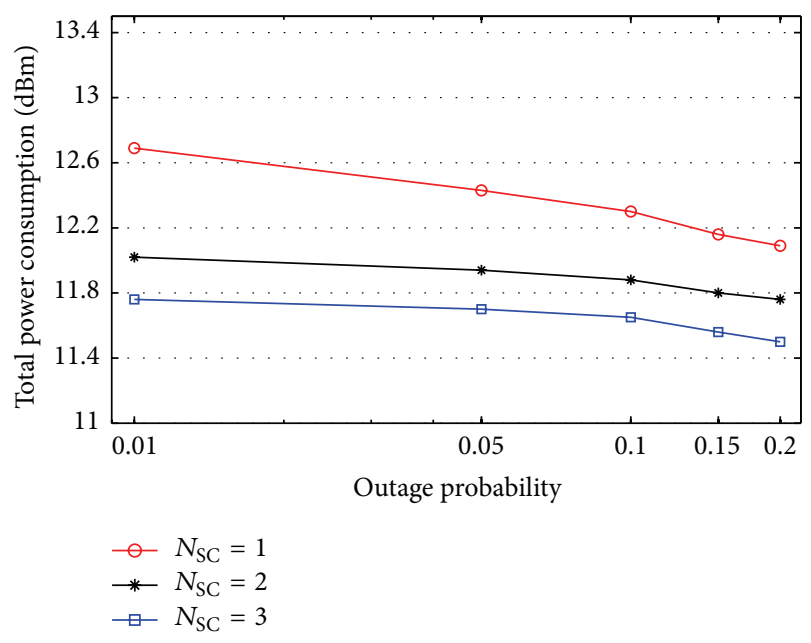

FIGURE 6: The average power consumption of the whole cell for different outage probability constraints.

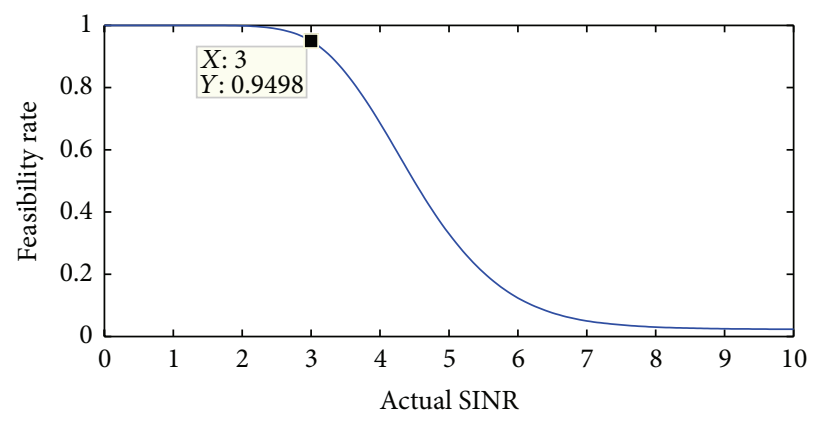

FIGURE 7: The feasible rate of the actual SINR satisfaction probabilities with $\delta_{k}=0.05$ and the given SINR constraint for each user is $\gamma_{k}=3$.

Figure 7 describes the feasible rate of the actual SINR satisfaction probabilities and the presented results are achieved from 40,000 random channel realizations. When $\delta_{k}=0.05$ and the given SINR constraint for each user is $\gamma_{k}=3$, we obtain the actual SINR satisfaction probabilities of one SUE in Figure 7. We find that $\operatorname{Pr}\left\{\Gamma_{k}>\gamma_{k}\right\}$ is $0.9498 \approx 1-\delta_{k}$ which meets our QoS outage probability constraints strictly.

Figure 8 shows the total number of iterations of the proposed algorithm with different $N$. In the presented results, each point is obtained from 10,000 random channel realizations. We find that the total number of iterations tends to be static with large number of $N$, for example, 3.5 times for $N_{\mathrm{SC}}=1$ and 3 times for $N_{\mathrm{SC}}=2$ or 3 . In all of our simulations, the maximum number of iterations is 25 when $\left\{N, N_{\mathrm{SC}}\right\}=\{16,1\}$. With the increasing of $N$, it will take fewer iterations to meet the stop criterion. When $N_{\mathrm{SC}}=1$, it always costs more iterations to meet the stop criterion. Hence, it is favorable to allocate more antennas at SCs in view of spending fewer times of iterations.

In summary, the above simulations show that the carefully chosen $\left\{N, N_{\mathrm{SC}}\right\}$ is acquired to maximize the energy efficiency of such two-tier HetNet. When $\left\{N, N_{\mathrm{SC}}\right\}$ is carefully 


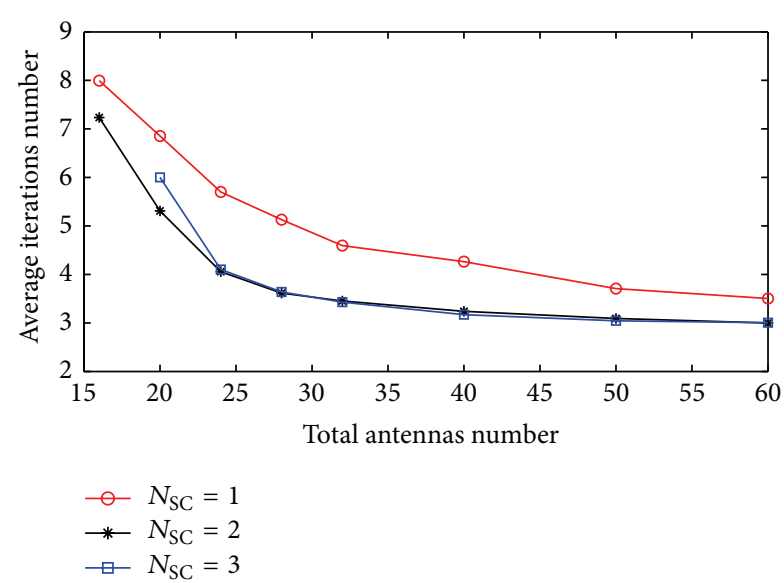

FIGURE 8: The number of iterations of the proposed algorithm with different numbers of $N$ and $N_{\mathrm{SC}}$.

chosen, the average energy consumption of the proposed pilot reuse scheme is larger than that of the full CSI scenario, and the gap between them is about $0.3 \mathrm{~dB}$. Over a tolerable range of the QoS outage probability, the power consumption of our scheme will stay nearly static. Specifically, when $N$ and $N_{\mathrm{SC}}$ are sufficient large or $\gamma_{k}$ is not too large, the proposed algorithm consumes less power than the perfect CSI scenario. Hence, the pilot reuse scheme and its associated beamforming algorithm are quite suitable for massive MIMO systems.

\section{Conclusion}

In this paper, we focus on a kind of two-tier networks, where massive MIMO is deployed in combination with small cell networks. However, it is not permissible to assign orthogonal pilot sequences for all the supported users due to the large number. We propose a pilot reuse scheme based on the unique architecture of this networks and find that a special kind of mixed CSI is yielded when using our pilot reuse scheme. Since mixed CSI may cause QoS outage the UEs, we formulate the downlink beamforming problem as a chance constraint programming. Then we decompose the original problem into simpler subproblems and provide an algorithm to combine these subproblems and solve them iteratively in a distributed fashion. The numerical results show that the average energy consumption of the proposed pilot reuse scheme and its associated beamforming algorithm is close to that of the perfect CSI scenario and the energy consumption is nearly static over a wide range of the QoS outage probabilities. The QoS requirements and QoS outage probability are also strictly controlled in our algorithm. Our scheme will not only greatly compress the number of pilot sequences, but also owns a high performance with low complexity, which is especially suitable for massive MIMO systems and will be crucially useful to put such multitier massive MIMO systems into practice.

\section{Appendix}

The power of the interference received by the $k$ th SUE can be written as follows:

$$
I_{k}=\mathbf{h}_{0, k}\left(\sum_{i \in \mathscr{M}} \mathbf{W}_{0, i}\right) \mathbf{h}_{0, k}^{H}, \quad \forall k \in \mathcal{S},
$$

where $\mathbf{W}_{0, i}=\mathbf{w}_{0, i} \mathbf{w}_{0, i}^{H}$.

Since all the transmit antennas of MBS are considered to be center collected, $\mathbf{h}_{0, k}$ can be rewritten as

$$
\mathbf{h}_{0, k}=\sqrt{d_{0, k}} \mathbf{z}_{0, k}
$$

where $\mathbf{z}_{0, k} \in \mathbb{C}^{N_{B S} \times 1}$ and the entries of $\mathbf{z}_{0, k}$ are the small scale fading factors of the $k$ th user with i.i.d. $\sim \mathscr{C} \mathscr{N}(0,1)$ variables. Then $I_{k}$ can be reformulated as follows:

$$
I_{k}=d_{0, k} \times \mathbf{z}_{0, k}\left(\sum_{i \in \mathscr{M}} \mathbf{W}_{0, i}\right) \mathbf{z}_{0, k}^{H}, \quad \forall k \in \mathcal{S} .
$$

Now we focus on a problem of calculating the CDF of an Hermitian quadratic form $\left(Y=\mathbf{z}_{0, k}\left(\sum_{i \in \mathscr{M}} \mathbf{W}_{0, i}\right) \mathbf{z}_{0, k}^{H}\right)$ in a Gaussian random vector $\mathbf{z}_{0, k}$. For a Gaussian random vector $\mathbf{z}_{0, k} \sim \mathscr{C} \mathcal{N}(0,1)$, the $\mathrm{CDF}$ of $Y=\mathbf{z}_{0, k} \mathbf{A} \mathbf{z}_{0, k}^{H}$ for some given $\mathbf{A}$ is given by [34]

$$
\begin{aligned}
F_{Y}(\tau) & =\operatorname{Pr}\left\{\mathbf{z}_{0, k} \mathbf{A} \mathbf{z}_{0, k}^{H} \leqslant \tau\right\} \\
& =\frac{1}{2 \pi} \int_{-\infty}^{\infty} \frac{e^{\tau(\beta+j \omega)}}{\beta+j \omega} \frac{1}{\operatorname{det}(\mathbf{I}+(\beta+j \omega) \mathbf{A})} d \omega
\end{aligned}
$$

for some $\beta>0$ such that $\mathbf{I}+\beta \mathbf{A}$ is positive definite. Here, $\mathbf{A}=\sum_{i \in \mathscr{M}} \mathbf{W}_{0, i}$. Since $\mathbf{W}_{i}, \forall i \in \mathscr{M}$, are positive semidefinite, $\mathbf{A}$ will also be positive semidefinite and the rank of $\mathbf{A}$ will be no larger than $m=|\mathscr{M}|$, where $|\mathscr{M}|$ means the cardinality of the set $\mathscr{M}$. It means that the matrix $\mathbf{A}$ has at most $m$ positive eigenvalues and the others are 0 . In most cases of communication systems, the rank of $\mathbf{A}$ is $m$ and the $m$ eigenvalues will be different with each other (as for the case where there exist the same eigenvalues, readers can refer to the procedure detailed in [34] and see the references therein).

Then, we the $\mathrm{CDF}$ of $Y$ can be reformulated as

$$
F_{Y}(\tau)=\frac{1}{2 \pi} \int_{-\infty}^{\infty} \frac{e^{\tau(\beta+j \omega)}}{\beta+j \omega} \prod_{i=1}^{m} \frac{1}{1+\lambda_{i}(\beta+j \omega)} d \omega,
$$

where $\lambda_{i}, i=1, \ldots, m$, are the nonzero eigenvalues of $\mathbf{A}$ in descending order.

By rewriting (A.5) as follows:

$$
\begin{aligned}
& \frac{e^{\tau(\beta+j \omega)}}{\beta+j \omega} \prod_{i=1}^{m} \frac{1}{1+\lambda_{i}(\beta+j \omega)} \\
& =e^{\tau(\beta+j \omega)}\left(\frac{1}{\beta+j \omega}+\sum_{i=1}^{m} \frac{\alpha_{i}}{1+\lambda_{i}(\beta+j \omega)}\right),
\end{aligned}
$$


where $\alpha_{i}=-\lambda_{i}^{m} / \prod_{l \neq i}\left(\lambda_{i}-\lambda_{l}\right)$, and using the property that

$$
\frac{1}{2 \pi} \int_{-\infty}^{\infty} \frac{e^{j \omega \tau}}{a+j \omega} d \omega=e^{-a \tau}, \quad \forall a>0,
$$

we can express (A.5) as

$$
F_{Y}(y)=1-\sum_{1}^{m} \frac{\lambda_{i}^{m-1}}{\prod_{l \neq i}\left(\lambda_{i}-\lambda_{l}\right)} e^{-y / \lambda_{i}}
$$

Hence, the power of the interference received by the $k$ th SUE can be rewritten as

$$
I_{k}=d_{0, k} F_{Y}^{-1}\left(1-\delta_{k}\right),
$$

where $F_{Y}^{-1}(\cdot)$ stand for the inverse function of (A.8).

\section{Conflict of Interests}

The authors declare that there is no conflict of interests regarding the publication of this paper.

\section{Acknowledgment}

This work has been supported by the National Natural Science Foundation of China (61171080).

\section{References}

[1] E. Bjornson, E. A. Jorswieck, M. Debbah, and B. Ottersten, "Multiobjective signal processing optimization: the way to balance conflicting metrics in 5G systems," IEEE Signal Processing Magazine, vol. 31, no. 6, pp. 14-23, 2014.

[2] E. G. Larsson, O. Edfors, F. Tufvesson, and T. L. Marzetta, "Massive MIMO for next generation wireless systems," IEEE Communications Magazine, vol. 52, no. 2, pp. 186-195, 2014.

[3] A. Ghosh, N. Mangalvedhe, R. Ratasuk et al., "Heterogeneous cellular networks: from theory to practice," IEEE Communications Magazine, vol. 50, no. 6, pp. 54-64, 2012.

[4] J. Hoydis, K. Hosseini, S. T. Brink, and M. Debbah, "Making smart use of excess antennas: massive MIMO, small cells, and TDD," Bell Labs Technical Journal, vol. 18, no. 2, pp. 5-21, 2013.

[5] J. Jose, A. Ashikhmin, T. L. Marzetta, and S. Vishwanath, "Pilot contamination problem in multi-cell TDD systems," in Proceedings of the IEEE International Symposium on Information Theory (ISIT '09), pp. 2184-2188, Seoul, Republic of Korea, July 2009.

[6] F. Fernandes, A. Ashikhmin, and T. L. Marzetta, "Inter-cell interference in noncooperative TDD large scale antenna systems," IEEE Journal on Selected Areas in Communications, vol. 31, no. 2, pp. 192-201, 2013.

[7] S. Jin, X. Wang, Z. Li, and K.-K. Wong, "Zero-forcing beamforming in massive MIMO systems with time-shifted pilots," in Proceedings of the IEEE International Conference on Communications (ICC '14), pp. 4801-4806, Sydney, Australia, June 2014.

[8] S. Jin, X. Wang, Z. Li, k.-k. Wong, Y. Huang, and X. Tang, "On massive MIMO zero-forcing transceiver using time-shifted pilots," IEEE Transactions on Vehicular Technology, 2015.

[9] E. Bjornson, E. G. Larsson, and M. Debbah, "Massive MIMO for maximal spectral efficiency: how many users and pilots should be allocated?", http://arxiv.org/abs/1412.7102.
[10] M. Li, S. Jin, and X. Gao, "Spatial orthogonality-based pilot reuse for multi-cell massive MIMO transmission," in Proceedings of the International Conference on Wireless Communications and Signal Processing (WCSP '13), pp. 1-6, October 2013.

[11] M. Mazrouei-Sebdani and W. A. Krzymień, "Massive MIMO with clustered pilot contamination precoding," in Proceedings of the 47th Asilomar Conference on Signals, Systems and Computers, pp. 1218-1222, November 2013.

[12] Y. Li, G. Xiqi, X. Xiang-Gen, M. Ni, and P. Yan, "Massive MIMO transmission with pilot reuse in single cell," in Proceedings of the IEEE International Conference on Communications (ICC '14), pp. 4783-4788, Sydney, Australia, June 2014.

[13] V. Chandrasekhar, M. Kountouris, and J. G. Andrews, "Coverage in tiered cellular networks with spatial diversity," in Proceedings of the IEEE Global Telecommunications Conference (GLOBECOM '09), pp. 1-6, Honolulu, Hawaii, USA, December 2009.

[14] Q. Li and W.-K. Ma, "Optimal and robust transmit designs for MISO channel secrecy by semidefinite programming," IEEE Transactions on Signal Processing, vol. 59, no. 8, pp. 3799-3812, 2011.

[15] H. Jing and A. L. Swindlehurst, "Robust secure transmission in MISO channels based on worst-case optimization," IEEE Transactions on Signal Processing, vol. 60, no. 4, pp. 1696-1707, 2012.

[16] K.-Y. Wang, T.-H. Chang, W.-K. Ma, A. M.-C. So, and C.-Y. Chi, "Probabilistic SINR constrained robust transmit beamforming: a bernstein-type inequality based conservative approach," in Proceedings of the 36th IEEE International Conference on Acoustics, Speech, and Signal Processing (ICASSP '11), pp. 3080-3083, Prague, Czech Republic, May 2011.

[17] S. Ma and D. Sun, "Chance constrained robust beamforming in cognitive radio networks," IEEE Communications Letters, vol. 17, no. 1, pp. 67-70, 2013.

[18] L. Vandenberghe and S. Boyd, "Semidefinite programming," SIAM Review, vol. 38, no. 1, pp. 49-95, 1996.

[19] Y. Wei, K. Taesoo, and S. Changyong, "Multicell coordination via joint scheduling, beamforming and power spectrum adaptation," in Proceedings of the IEEE Conference on Computer Communications (INFOCOM '11), pp. 2570-2578, Shanghai, China, 2011.

[20] D. W. K. Ng, E. S. Lo, and R. Schober, "Energy-efficient resource allocation in OFDMA systems with large numbers of base station antennas," IEEE Transactions on Wireless Communications, vol. 11, no. 9, pp. 3292-3304, 2012.

[21] T. L. Marzetta, "How much training is required for multiuser MIMO?" in Proceedings of the 40th Asilomar Conference on Signals, Systems, and Computers (ACSSC '06), pp. 359-363, Pacific Grove, Calif, USA, November 2006.

[22] K. Guo, Y. Guo, G. Fodor, and G. Ascheid, "Uplink power control with MMSE receiver in multi-cell MU-massive-MIMO systems," in Proceedings of the IEEE International Conference on Communications (ICC '14), pp. 5184-5190, Sydney, Australia, June 2014.

[23] Q. N. Hien, M. Matthaiou, and E. G. Larsson, "Massive MIMO with optimal power and training duration allocation," IEEE Wireless Communications Letters, vol. 3, no. 6, pp. 605-608, 2014.

[24] A. Ashikhmin and T. Marzetta, "Pilot contamination precoding in multi-cell large scale antenna systems," in Proceedings of the IEEE International Symposium on Information Theory (ISIT '12), pp. 1137-1141, IEEE, Cambridge, Mass, USA, July 2012. 
[25] S. Boyd and L. Vandenberghe, Convex Optimization, Cambridge University Press, New York, NY, USA, 2004.

[26] J. F. Sturm, "Using SeDuMi 1.02, a MATLAB toolbox for optimization over symmetric cones," Optimization Methods and Software, vol. 11, no. 1, pp. 625-653, 1999.

[27] C. Wang and R. D. Murch, "Adaptive downlink multi-user MIMO wireless systems for correlated channels with imperfect CSI," IEEE Transactions on Wireless Communications, vol. 5, no. 9, pp. 2435-2446, 2006.

[28] Y. Xinping and E. K. S. Au, "User scheduling for heterogeneous multiuser MIMO systems: a subspace viewpoint," IEEE Transactions on Vehicular Technology, vol. 60, no. 8, pp. 4004-4013, 2011.

[29] Q. Sun, Y. Xue, Y. Zhang, S. Jin, X. Li, and X. Gao, "User assignment for MU-MIMO downlink system based on Munkres algorithm," in Proceedings of the International Conference on Wireless Communications and Signal Processing (WCSP '13), pp. 1-6, October 2013.

[30] C. Shanzhi and Z. Jian, "The requirements, challenges, and technologies for $5 \mathrm{G}$ of terrestrial mobile telecommunication," IEEE Communications Magazine, vol. 52, no. 5, pp. 36-43, 2014.

[31] G. Auer, V. Giannini, C. Desset et al., "How much energy is needed to run a wireless network?" IEEE Wireless Communications, vol. 18, no. 5, pp. 40-49, 2011.

[32] R. Muharar, R. Zakhour, and J. Evans, "Base station cooperation with limited feedback: a large system analysis," in Proceedings of the IEEE International Symposium on Information Theory (ISIT '12), pp. 1152-1156, Cambridge, Mass, USA, July 2012.

[33] L. C. Godara, Handbook of Antennas in Wireless Communications, CRC Press, 2001.

[34] T. Y. Al-Naffouri and B. Hassibi, "On the distribution of indefinite quadratic forms in gaussian random variables," in Proceedings of the IEEE International Symposium on Information Theory (ISIT '09), pp. 1744-1748, Seoul, Republic of Korea, July 2009. 

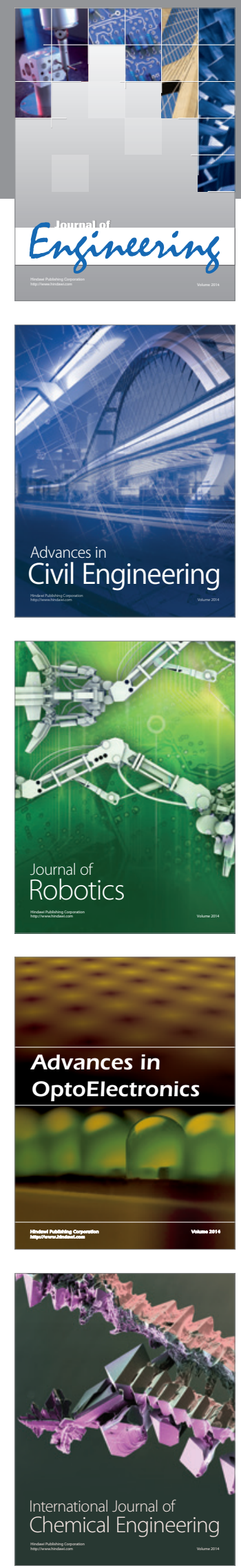

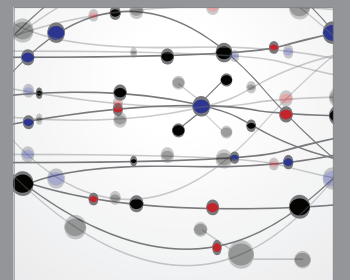

The Scientific World Journal
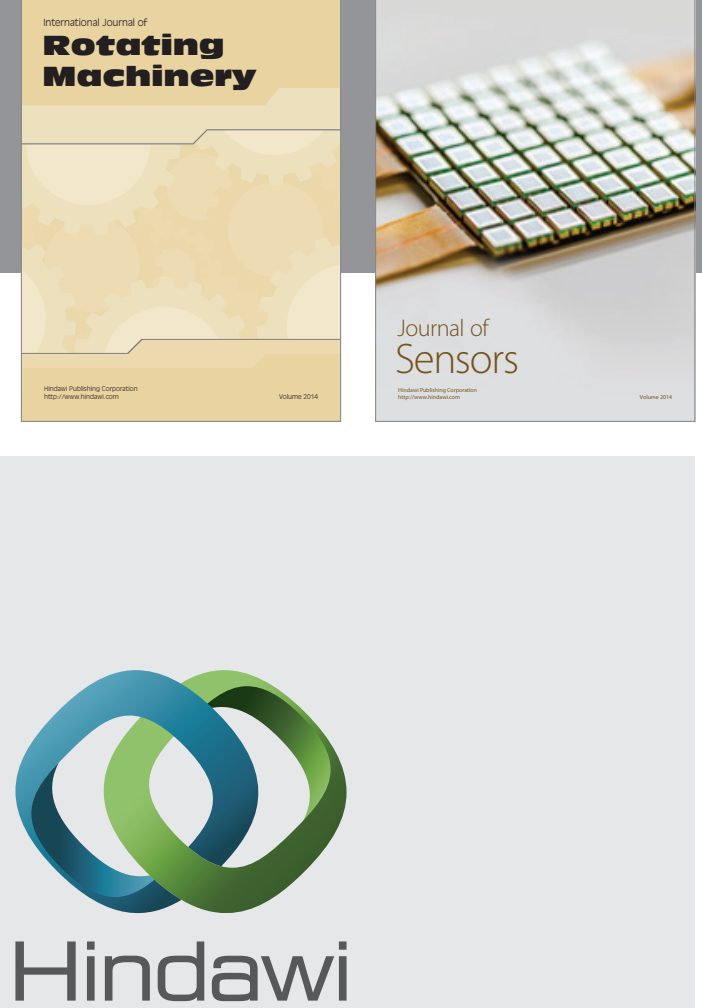

Submit your manuscripts at http://www.hindawi.com
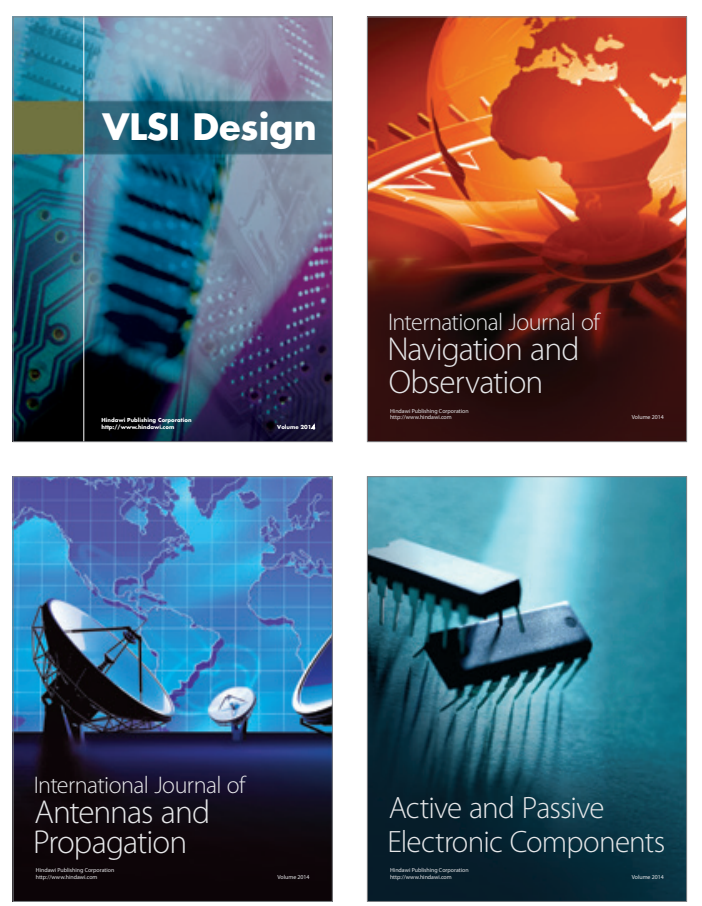
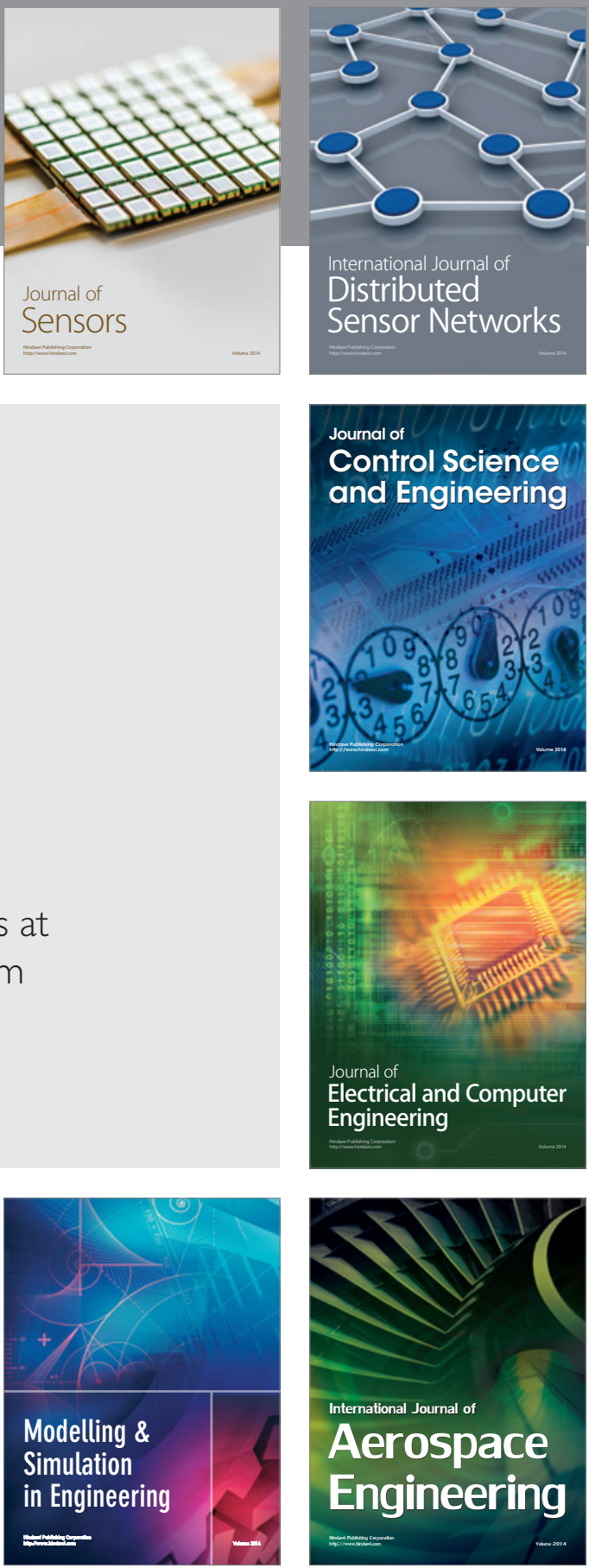

Journal of

Control Science

and Engineering
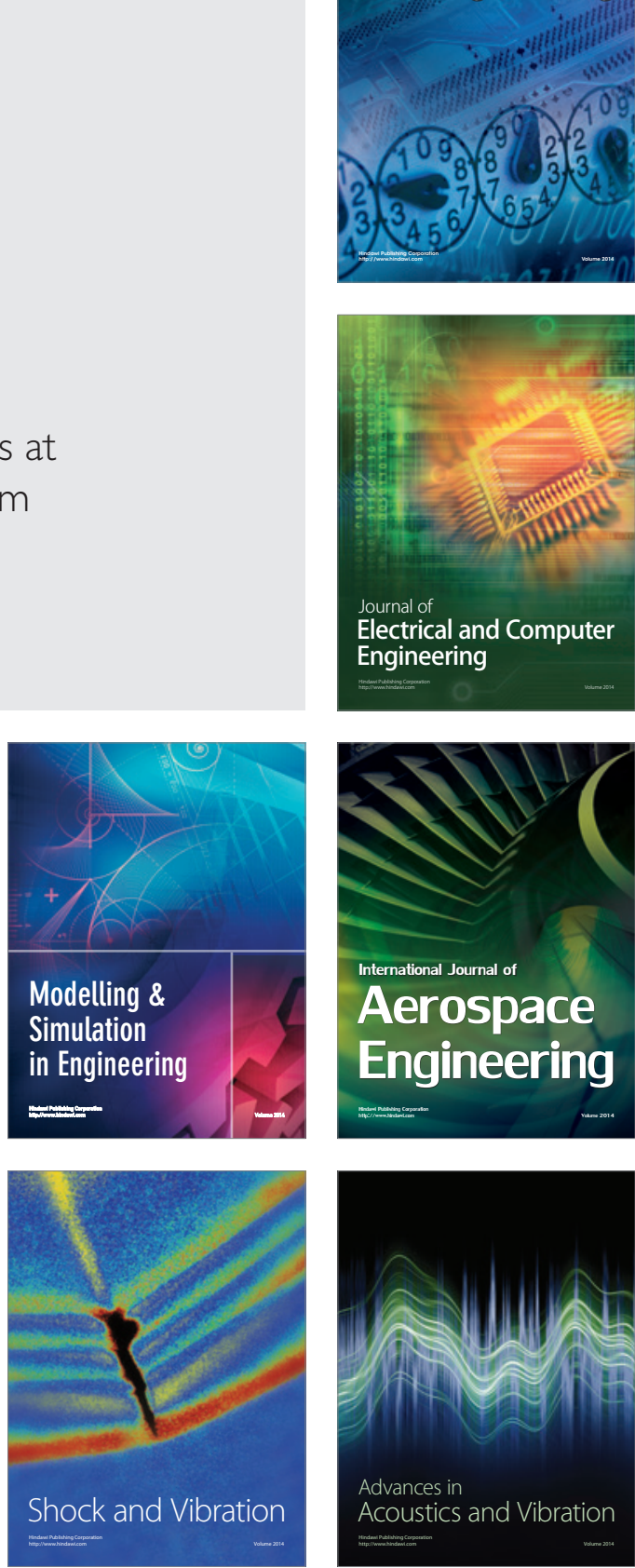